\title{
Modeling heterogeneity and memory effects on the kinetic roughening of silica film grown by chemical vapor deposition
}

\author{
Fernando Ojeda, ${ }^{1, *}$ Rodolfo Cuerno, ${ }^{2, \dagger}$ Roberto Salvarezza, ${ }^{3, \dagger}$ Fernando Agulló-Rueda, ${ }^{1,}$ and Luis Vázquez, ${ }^{1, \|}$ \\ ${ }^{1}$ Instituto de Ciencia de Materiales de Madrid (CSIC), Cantoblanco, E 28049 Madrid, Spain \\ ${ }^{2}$ Departamento de Matemáticas \& GISC, Universidad Carlos III de Madrid, Avenida de la Universidad 30, E 28911 Leganés, Spain \\ ${ }^{3}$ INIFTA, Sucursal 4, Casilla de Correo 16, (1900) La Plata, Argentina
}

\begin{abstract}
We present discrete and continuum models to describe previous experiments on growth of chemical vapor deposited silica film at $611 \mathrm{~K}$ and $723 \mathrm{~K}$ [F. Ojeda et al., Phys. Rev. Lett. 84, 3125 (2000)]. Silica film deposited at $723 \mathrm{~K}$ show larger surface roughness already from the early stages of growth, a fact that was not explained by a previously proposed phenomenological stochastic equation for the interface height. This larger surface roughness and the concomitant development of higher local slopes seem to be correlated with the Kardar Parisi Zhang asymptotic scaling observed for the high temperature conditions. Here, we explain these features on the basis of surface heterogeneity and short range memory effects, which are assessed for our experimental system through spectroscopic measurements. By incorporating these effects into a random depo sition model and related Langevin equations with correlated noise, we are able to account for the full set of experimental observations.
\end{abstract}

DOI: 10.1103/PhysRevB.67.245416

PACS number(s): 68.35.Ct, 81.15.Gh, 64.60.Ht, 05.10.Gg

\section{INTRODUCTION}

Low-pressure chemical vapor deposition (LPCVD) is one of the most frequently used techniques for thin-fil production in several industrial applications. ${ }^{1}$ Silicon dioxide film have been extensively used in very large scale integrated devices. Knowledge and eventual control of the morphology of silica film produced by CVD become very important as device dimensions are being continuously reduced. In particular, this control becomes critical when conformal growth (i.e., good step coverage) is required. In typical LPCVD processes, the precursor species arrive at the growing interface following random-walk trajectories through the stagnant layer. This fact implies that protrusions at the interface tend to receive more incoming particles than depressions, ${ }^{2}{ }^{5}$ giving place to shadowing effects among the various surface features. These effects usually lead to unstable interfaces characterized by large surface roughness. On the other hand, it is known ${ }^{4,6}$ that conformal growth is promoted for those systems displaying low surface reaction kinetics (low sticking probability in the language of Monte Carlo models of CVD, Ref. 6). Specificall , it is accepted that the mechanism inducing conformal growth is reemission of the precursor (i.e., depositing) species due to a low sticking probability. For micrometer structures onto which conformal growth is required, much is currently known on the interplay between effects such as shadowing and low sticking probability. However, when considering conformal growth on submicron features, much less is known on the relative balance between these mechanisms, and there is not sufficien control yet on growth conditions at these length scales. Therefore, due to the ever shrinking dimensions of technological devices, detailed study of CVD silica fil morphology at submicron scales becomes necessary.

During the last decade or so, the study of thin-fil growth dynamics has attracted considerable interest. ${ }^{7,8}$ Many of these studies, both theoretical and experimental, have been performed within the framework of the dynamic scaling ansatz. ${ }^{9}$ Briefl, this is a statement on the time and scale invariance of the fil surface roughness or surface width, which is define as $\sigma(t)=\left(\left(1 / L^{2}\right)\left\langle\Sigma_{\mathrm{r}}[h(\mathbf{r}, t)-h(t)]^{2}\right\rangle\right)^{1 / 2}$, where $h(\mathbf{r}, t)$ describes the value of the surface height at time $t$ above position $\mathbf{r}$ on a substrate plane of lateral dimension $L$. In what follows, an overline will denote spatial average across a given sample and the brackets $\langle\ldots\rangle$ will stand for averages over different samples. For many surfaces evolving under nonequilibrium conditions, it is observed ${ }^{7,8}$ that the roughness increases with deposition time as $\sigma \sim t^{\beta}$, with $\beta$ being termed the growth exponent. Surface features across the substrate plane, as measured by the characteristic lateral correlation length $\xi$ also coarsen with time as $\xi \sim t^{1 / z}$, with $z$ an independent dynamic exponent. Once $\xi$ becomes larger than $L$, the roughness saturates into a stationary value that scales as $\sigma \sim L^{\alpha}$, with $\alpha=z \beta$ being the roughness exponent.

Scanning probe microscopies, in particular, atomic force microscopy (AFM), are rather well adapted to quantitatively analyze the surface morphology. AFM allows for the measurement of the surface roughness over a wide range of length scales. This is important as it allows for the assessment of the scale invariance of the surface features, and even distinguishing different scaling regimes with the corresponding crossover regions, which may exist for a given system. Thus, from the AFM data it is possible to obtain the values of the three scaling exponents independently and compare them with those predicted by the different growth models. ${ }^{7,8} \mathrm{We}$ have followed this procedure recently $y^{10,11}$ for experiments of CVD silica fil growth on $\mathrm{Si}(100)$ from silane/oxygen gas mixtures at two deposition temperatures, namely, $T=611 \mathrm{~K}$ and $723 \mathrm{~K}$, and keeping a constant deposition rate of 20 $\mathrm{nm} / \mathrm{s}$. Under these conditions, the system presents high and low sticking probabilities, respectively, as has been reported in Ref. 12. Namely, an increase in temperature from $573 \mathrm{~K}$ to 
$723 \mathrm{~K}$ was experimentally observed to induce a decrease in the average sticking probability $s$ from $s=0.5$ down to $s$ $=0.08$. Summarizing our previous results in Ref. 10, we found that the fil surface morphology resulted from the competition between stabilizing effects, such as surface diffusion and lateral growth, which is the fingerprin of KardarParisi-Zhang (KPZ) (Ref. 13) scaling behavior, and unstabilizing effects, originating in shadowing effects and the random arrival of the incoming particles. For the lowtemperature films the surface roughness increases unboundedly, within the temporal window considered, due to the shadowing effects. On the contrary, for the high-temperature film the system eventually stabilizes into a state displaying the asymptotic properties of the KPZ universality class. In fact, the surface roughness for $T=723 \mathrm{~K}$ approached saturation for very long deposition times. The physical origin of the asymptotic KPZ stabilization for the high-temperature silica film thus seems to be in the low sticking probability: under low sticking conditions, the shadowing effects characteristic of the CVD processes are counterbalanced by a more even redistribution of the incoming particles across the fil surface through reemission processes. As the corresponding observed scaling behavior is KPZ, these processes actually promote lateral growth. All these effects are accounted for in the following continuum stochastic model we proposed phenomenologically in Ref. 10 to describe the low-pressure CVD experiments:

$$
\begin{aligned}
& \frac{\partial h}{\partial t}=v-K \nabla^{4} h+\epsilon \frac{\theta}{\theta}+\frac{\lambda}{2}(\nabla h)^{2}+\eta(x, t), \\
& \left\langle\eta(x, t) \eta\left(x^{\prime}, t^{\prime}\right)\right\rangle=2 D \delta\left(x-x^{\prime}\right) \delta\left(t-t^{\prime}\right) .
\end{aligned}
$$

In Eq. (1a), the left-hand side represents the local velocity of the growing interface. The firs term on the right-hand side (rhs) is the average growth rate for a fla interface, the second term represents relaxation by surface diffusion due to curvature gradients, ${ }^{14}$ the third term represents geometric shadowing effects, ${ }^{15,16} \theta$ being the local exposure angle and $\theta$ the spatial average of $\theta$, and the fourth nonlinear term accounts for growth along the local normal direction ${ }^{13}$ (lateral growth). The term $\eta(x, t)$ in Eqs. (1) is a zero-average Gaussian white noise that represents microscopic fluctua tions in the attachment-detachment events at the growing surface. The parameters $K, \epsilon, \lambda$, and $D$ are positive constants. ${ }^{10}$

Simulations of model (1) for a one-dimensional substrate the asymptotic properties of two-dimensional substrates are prohibitive for numerical simulations due to the computational requirements of the shadowing term ${ }^{16}$ reproduced qualitatively the observed behavior for both deposition temperatures, assuming that the KPZ coefficien $\lambda$ was larger at $723 \mathrm{~K}$. There is, however, one important point that remained unaccounted for by model (1); namely, the films grown under low sticking conditions (high temperature) were rougher, i.e., the numerical value of $\sigma$ was larger than those deposited under high sticking conditions (low temperature). We should emphasize that this difference occurs al- ready from the very beginning of the deposition experiments. This counterintuitive feature the film produced under a more stable dynamics are rougher cannot be explained by model (1) since the second and fourth terms in Eq. (1a), having stabilizing effects, are actually more important under the high-temperature deposition conditions. ${ }^{10}$ Specificall, the fourth derivative term describes surface diffusion, which operates on relatively short length scales. Therefore, its influenc on the surface morphology should be more clearly appreciated in the early stages of growth. This would cause the low-temperature film to be rougher than the hightemperature set, at least at short growth times. However, our observations contradict this expectation, justifying our previous statement that surface diffusion cannot be held responsible for the larger roughness at a higher temperature. Another physical mechanism that might correlate with the different roughness values for the two temperature conditions is the difference in their grain nucleation rates. However, for our system this influenc is likely to be overcome by the low sticking probability effects at high temperatures. Even if the grain nucleation rate were smaller at high $T$ (which is doubtful since the flu of species is higher in order to achieve the same growth rate, common for both temperature conditions), the lower sticking probability is expected to counteract the potential excess roughness induced by this effect.

In this paper, we provide an explanation for the observed behavior of the roughness that, beyond accounting for the same scaling properties as model (1), is able to reproduce the larger value of $\sigma$ for the more stable growth condition. The origin of this feature is traced back to chemical heterogeneity at the surface of the growing film which we assess by spectroscopic measurements. In brief, the existence of surface species at which growth is more efficien than others, combined with memory effects which occur during silica CVD growth, enhances surface roughness. This nonuniformity among surface sites is stronger at high temperatures.

We firs study the chemical and morphological properties of the surface the latter as measured by AFM in Secs. II and III. After this, we devote Secs. IV and V to propose a generalized model that is able to reproduce the full set of observations. Specificall, in Sec. IV we demonstrate by means of the simplest discrete growth model [namely, random deposition ${ }^{7}(\mathrm{RD})$ ] how surface heterogeneity coupled to memory effects does lead to enhanced roughness. Based on results in this section, we then generalize in Sec. V the con tinuum model (1) in order to account for these effects. We will show that a continuum equation such as Eqs. (1), but in which the noise has short-range temporal correlations $^{17}$ rather than being white as in Eq. (1b) accounts for the increased roughness maintaining the same observed scaling behavior. Physically, the correlations of the noise arise from the coexistence of memory effects and surface heterogeneity during silica CVD growth. ${ }^{18,19}$ It is important to stress that these new finding do not invalidate our previous results concerning the CVD silica growth evolution, rather they contribute to a more detailed understanding of such a complex growth process. 

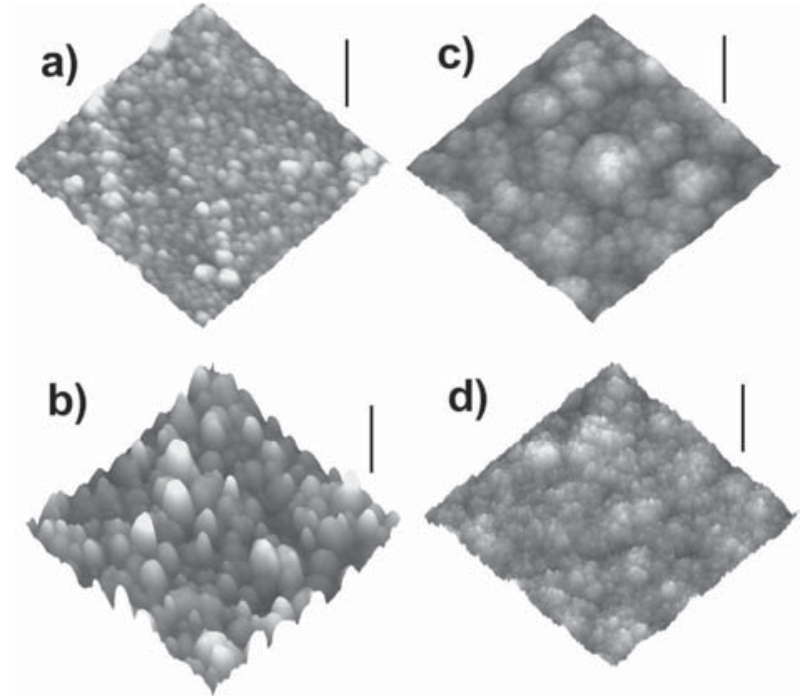

FIG. 1. $500 \times 500 \mathrm{~nm}^{2}$ AFM images of amorphous $\mathrm{SiO}_{2}$ film deposited for $20 \mathrm{~min}$ at $611 \mathrm{~K}$ (a) and $723 \mathrm{~K}$ (b). The bar indicates $80 \mathrm{~nm}$. Panels (c) and (d) are $14 \times 14 \mu \mathrm{m}^{2}$ AFM images taken after two days deposition for $T=611 \mathrm{~K}$ and $T=723 \mathrm{~K}$, respectively. In these two panels, the bar indicates $1 \mu \mathrm{m}$.

\section{EXPERIMENT}

Amorphous $\mathrm{SiO}_{2}$ film were grown at a temperature $T$ $=723 \mathrm{~K}$ (low sticking condition) and $T=611 \mathrm{~K}$ (high sticking condition) on $\mathrm{Si}(100)$ substrates in a hot wall, horizontal, low-pressure tubular CVD reactor. The precursor gases were silane (diluted at $2 \%$ in nitrogen, $99.999 \%$ purity) and oxygen $(99.9992 \%$ purity), with an oxygen/silane ratio equal to 20 and a total gas flo rate $R_{g}$ of 50 and $250 \mathrm{sccm}$, respectively. The chamber pressure was 1.4 torr. The fil thickness increased linearly with deposition time at a constant growth rate of $20 \mathrm{~nm} / \mathrm{min}$ for both temperature conditions. Films were deposited in the range $5 \mathrm{~min} \leqslant t \leqslant 2$ days in order to be able to observe the asymptotic growth behavior. It should be noted that due to the many processes occurring in the CVD experiment, a precision better than $1 \mathrm{~min}$ in deposition time is unreachable with our experimental setup. The surface morphology was characterized by AFM (Nanoscope IIIa from Digital Instruments, CA), operating in the tapping mode at ambient conditions over length scales ranging from $50 \mathrm{~nm}$ up to $50 \mu \mathrm{m}$. Silicon cantilevers were employed. Films were also characterized by infrared (IR) and Raman spectroscopies. Unpolarized Raman spectra were taken with a Renishaw Ramascope 2000 microspectrometer and an $\mathrm{Ar}^{+}$ laser $(514.5 \mathrm{~nm})$ for excitation.

\section{MORPHOLOGICAL AND CHEMICAL CHARACTERIZATION}

Figure 1 shows $500 \times 500 \mathrm{~nm}^{2}$ AFM images of our silica film grown for $20 \mathrm{~min}$ at $611 \mathrm{~K}$ [Fig. 1(a)] and $723 \mathrm{~K}$ [Fig. 1(b)]. The deposit is formed by small rounded grains $30 \quad 60$ $\mathrm{nm}$ in size. Indeed, these images indicate that the surface fil roughness $\sigma$ is larger for the high-temperature set al- ready at short deposition times. This difference persists up to very long deposition times, as can be appreciated in Figs. 1(c) and 1(d), that show $14 \times 14 \mu \mathrm{m}^{2}$ AFM images taken after two days of deposition. The differences between the fil morphologies obtained for the two temperature conditions are clear, in particular, the protrusions of micrometer size appearing only for the $611-\mathrm{K}$ set of films However, the $723-\mathrm{K}$ film still have larger roughness values than the 611-K sets.

Figures 2(a) 2(c) show the results for the main morphological characterization of the high- and low-temperature films First, the plot of $\sigma$ as a function of time [Fig. 2(a)] shows quantitatively that the surface roughness is larger for the $723 \mathrm{~K}$ film (low sticking set) than for the $611 \mathrm{~K}$ ones within the whole temporal window studied. This effect is also observed in Fig. 2(b), which displays the power spectral density (PSD) of the surface morphology for film deposited after two days for both temperature conditions. The PSD, say $S$, define as

$$
S(k, t)=\frac{4 \pi^{2}}{L^{2}}\langle h(\mathbf{k}, t) h(-\mathbf{k}, t)\rangle,
$$

where $h(\mathbf{k}, t)$ is the two-dimensional Fourier transform of $h(\mathbf{r}, t)-h(t)$ and $\mathbf{k}$ is the wave vector of modulus $k=|\mathbf{k}|$. For a rough surface, the PSD function behaves generically as $^{7,8} S(k) \sim k^{-2 \alpha-2}$; hence from a logarithmic plot of the $S$ vs $k$, one can extract the value of the roughness exponent at the corresponding length scales. Figure 2(b) thus shows the scaling behavior at long times for the two temperature conditions. Films deposited at 611 K [lower panel of Fig. 2(b)] display an initial scaling region dominated by surface diffusion relaxation processes $(\alpha \simeq 1)$ with an asymptotic unstable $(\alpha \simeq 0.7)$ scaling behavior dominated by shadowing effects. ${ }^{10}$ On the other hand, film grown at $723 \mathrm{~K}$ [upper panel of Fig. 2(b)] display also an initial scaling region due to surface diffusion effects followed by an unstable regime. However, the asymptotic regime results into KPZ scaling behavior ( $\alpha \simeq 0.4, \beta \simeq 0.25$ ). It is worth mentioning that the identificatio of the various growth regimes in Ref. 10 was done after obtaining the three $(\alpha, \beta$, and $1 / z)$ scaling exponents independently. ${ }^{11}$

We have also analyzed the average slope across the fil surface, $m$, for both sets as deposition proceeds. Specificall, the surface slope was computed at different sites on the AFM scan for each sample from linear fit by least squares over a fixe window size $\ell$ and was then spatially averaged in order to obtain the average slope $m$. Figure 2(c) shows the evolution of $m$ with time for $\ell=200 \mathrm{~nm}$. It can be seen that $m$ is also always larger for the high-temperature condition already from the early stages of growth. In principle, the observed difference in surface slope is directly related to that in surface roughness, since surface diffusion effects are quite similar at both temperatures. Moreover, the larger surface slope observed for the high-temperature set appears to be correlated with the KPZ behavior observed in the long temporal and spatial scales corresponding to this set of films ${ }^{10}$ Note that actually the KPZ nonlinearity is an increasing function of $m$. These phenomena seem reminiscent of the stabiliza- 

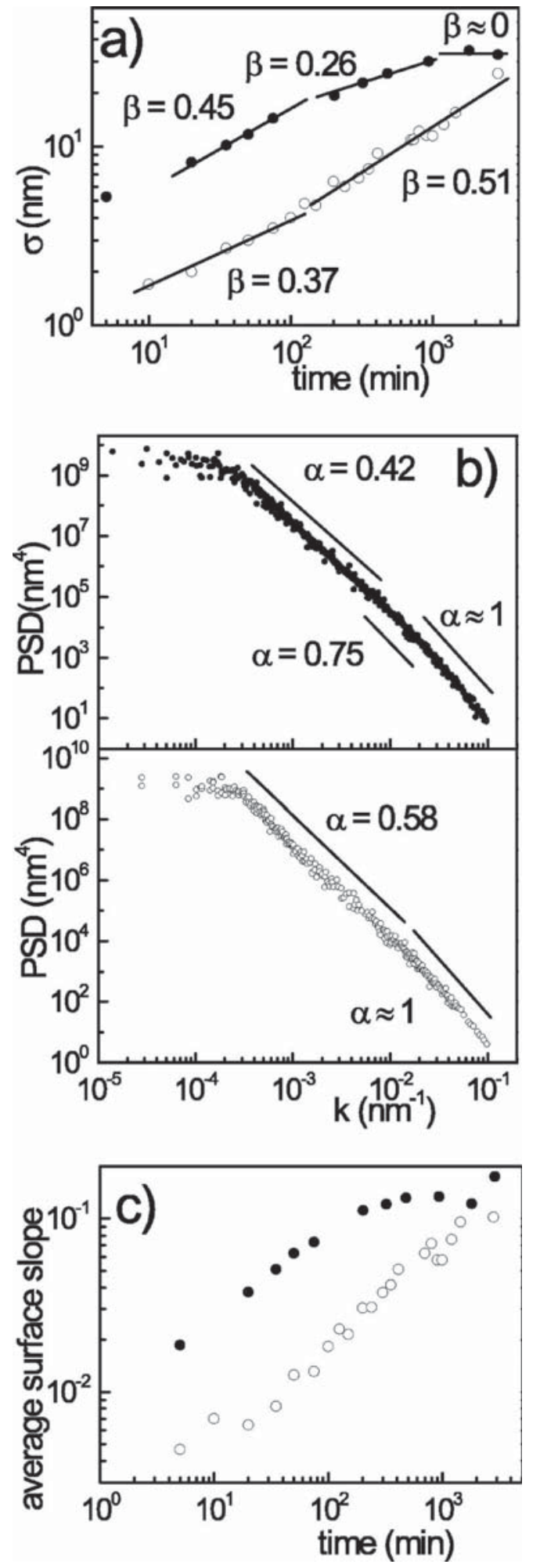

FIG. 2. (a) Time evolution of the surface roughness $\sigma(t)$ for the $611 \mathrm{~K}$ film (O) and $723 \mathrm{~K}$ film (@). (b) PSD curves from AFM images of the $\mathrm{SiO}_{2}$ surface deposited after two days deposition at $611 \mathrm{~K}(\bigcirc)$ and $723 \mathrm{~K}(\bigcirc)$. The straight lines in (a) and (b) indicate the relevant exponent values for the various scaling regimes. (c) Time evolution of the average slope $\bar{m}(t)$ computed using $\ell$ $=200 \mathrm{~nm}$ for the $611 \mathrm{~K}$ film $(\bigcirc)$ and $723 \mathrm{~K}$ film
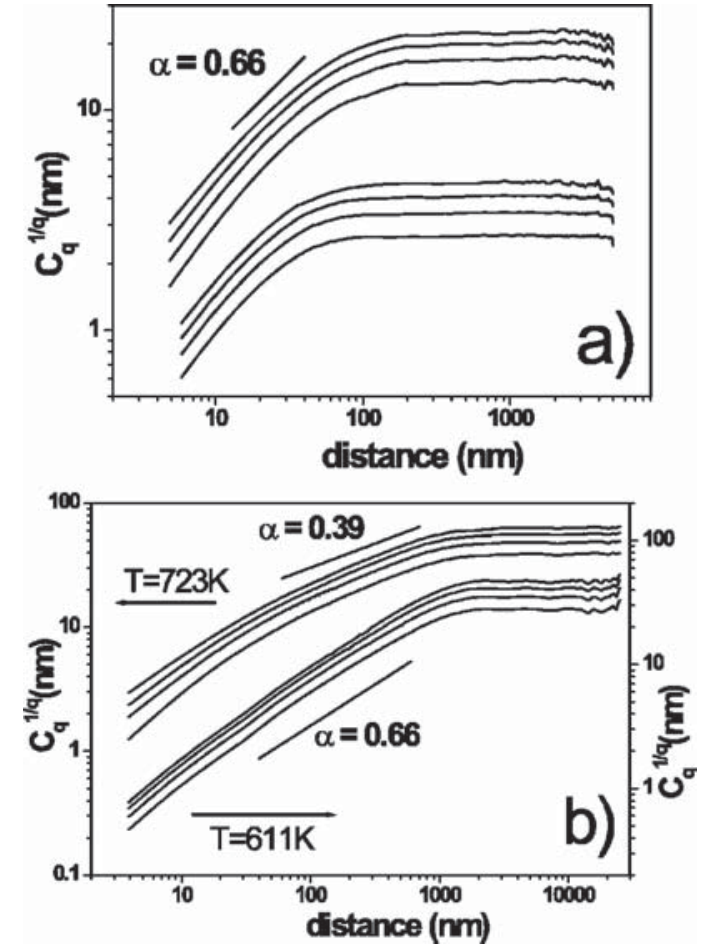

FIG. 3. Logarithmic plots of the $q$ th root of the moments of the height difference distribution $C_{q}(r, t)$ [Eq. (3)] for several values of $q$, computed for the two temperature conditions, and times $t$ $=35 \min$ (a) and $t=2$ days (b). In each panel, the four uppermost curves correspond to the $T=723 \mathrm{~K}$ set, in the sequence $q$ $=1,2,3,4$, bottom to top, and the four lowermost curves to the con dition $T=611 \mathrm{~K}$, in an analogous sequence. Straight lines are shown for reference having slopes, as indicated, that correspond to relevant values of the roughness exponent $\alpha$.

tion by a KPZ nonlinearity of dynamical instabilities occurring in the Kuramoto-Sivashinsky equation and its noisy generalizations. ${ }^{20,21}$ In any case, it seems that the elucidation of the origin of the increased surface roughness, and accordingly of the surface slope, is important in order to provide a full description of our system.

In order to provide a complete characterization of the fil morphology beyond the mean and the variance of the surface height, we have computed various moments of the heightdifference distribution, namely, the values of the function

$$
C_{q}(r, t)=\left\langle\left|h\left(\mathbf{r}_{1}, t\right)-h\left(\mathbf{r}_{2}, t\right)\right|^{q}\right\rangle
$$

for different values of $q$, where $r=\left|\mathbf{r}_{1}-\mathbf{r}_{2}\right|$. For multiaffin surfaces, each of these moments scales as $C_{q}(r) \sim r^{q \alpha_{q}}$ with an exponent $\alpha_{q}$ that is different for each value of $q .^{7}$ It is only for truly self-affin surfaces that $\alpha \equiv \alpha_{q}$ has a constant value independent of $q$, and thus $C_{q}(r) \sim r^{q \alpha}$. In Fig. 3, we show the results obtained for the $q$ th root of functions (3) $(q=1,2,3,4)$ for the film deposited at both temperatures, at times $t=35 \mathrm{~min}$ [Fig. 3(a)] and $t=2$ days [Fig. 3(b)]. Note that, once taken the $q$ th root, the different moments are parallel to one another in these logarithmic plots, meaning that our experimental surfaces are truly self-affin objects in the sense define above. This is an important consistency check 




FIG. 4. Raman spectra of $1.0 \mu \mathrm{m}$ thick amorphous $\mathrm{SiO}_{2}$ film deposited at $611 \mathrm{~K}$ (top curve) and $723 \mathrm{~K}$ (bottom curve). Four peaks have been marked: three of them correspond to two , three , and four membered siloxane rings, $R_{2}$ at $876 \mathrm{~cm}^{-1}, R_{3}$ at $605 \mathrm{~cm}^{-1}$, and $R_{4}$ at $502 \mathrm{~cm}^{-1}$, respectively; the remaining one at $519 \mathrm{~cm}^{-1}$ corresponds to the silicon substrate.

for our morphological analysis, to be considered again later when, we consider theoretical models of our experimental system.

As stated before, a mechanism which is potentially responsible for the morphological differences between the two temperature conditions studied is the variation with temperature of the sticking probability, $s$. In order to further investigate the implications of this change, we have analyzed the fil structure by means of IR and Raman spectroscopies. Physically, $s$ can be related both to the surface coverage and the chemical activity of reactive sites at the growing fil surface. For our system these sites are mainly associated with hydrogenated groups, such as $\mathrm{SiOH}$ and $\mathrm{SiH}$, and with strained siloxane ( $\mathrm{SiOSi}$ ) groups, rather than with the less reactive relaxed siloxane groups. ${ }^{22}{ }^{24}$ However, the activity of these reactive sites is different. For instance, the $\mathrm{SiH}$ bond weakness makes it a preferentially reactive site for fil growth as compared with $\mathrm{SiOH}$ groups. Consequently, it is necessary to study the relative concentrations of the various reactive sites on both sets of films We have quantifie the concentration of $\mathrm{SiH}$ and $\mathrm{SiOH}$ groups by IR spectroscopy (see Fig. 2 of Ref. 25) for both sets of films The most important result is the one concerning the $\mathrm{SiH}$ content, which is at least one order of magnitude higher for the $611-\mathrm{K}$ film than for the $723-\mathrm{K}$ samples. Besides, for the fil grown at low temperature, the Raman spectrum [Fig. 4] shows a clear peak, $R_{2}$ which has been related to the concentration of highly strained two-membered siloxane rings. ${ }^{26}$ These sites are expected to have a reactivity ten times that of the $\mathrm{SiOH}$ groups under typical CVD conditions. ${ }^{27}$ The present data allow us to conclude that the film grown at $611 \mathrm{~K}$ feature a higher content of strained ( $\mathrm{SiH}$, strained siloxane) groups than the $723-\mathrm{K}$ set. Thus, these film should have a higher internal stress than the high-temperature films which is consistent with the higher refraction index of the former. ${ }^{25}$ If the internal stresses were relevant to the morphological features, a higher value would induce larger roughness, thus the film
TABLE I. Ratio of the concentration of the different reactive sites between the high and low temperature films as measured by IR and Raman spectroscopies. $R_{2}$ and $R_{3}$ correspond to two and three membered siloxane rings, respectively.

\begin{tabular}{lcc}
\hline \hline Site & $\begin{array}{c}\text { Concentration ratio } \\
{[723 \mathrm{~K}] /[611 \mathrm{~K}]}\end{array}$ & Spectroscopy \\
\hline$[\mathrm{SiH}]$ & $>11$ & IR \\
{$[\mathrm{SiOHSi}]$} & 1.15 & IR \\
{$\left[R_{3}\right]$} & 1.34 & Raman \\
{$\left[R_{2}\right]$} & $>5$ & Raman \\
\hline \hline
\end{tabular}

grown at $611 \mathrm{~K}$ should be rougher than those produced at $723 \mathrm{~K}$, contrary to what is observed. Hence, internal stress can be overlooked as a main physical mechanism in order to account for the behavior observed in our system.

In Table I, we summarize our measurements by IR and Raman spectroscopies for the ratios of the relative concentrations of the different reactive sites between both sets of samples. ${ }^{25}$ It is clear that the relative concentrations of the various reactive sites are always higher for the lowtemperature film than for the high-temperature ones, in agreement with the reported ${ }^{12}$ values of the average sticking probability. From these data it is evident that both sets of film have both active and passive (namely, less active) sites, and that the high-temperature set presents a lower concentration of active sites than the low-temperature set. On the other hand, it has been recently reported ${ }^{18,19}$ for silica CVD film deposited from tetraethylorthosilicate (TEOS)/ozone precursors that there exist memory effects during growth that influ ence the fina fil morphology, in particular, the fil surface roughness. These memory effects are related to the existence of surface sites of different activities for deposition, and to the persistence in time of the activity properties for a given site on the substrate plane. Thus, the growth mode becomes site dependent. In the experiments of Refs. 18 and 19, the surface distribution of active sites was strongly linked to the type of initial substrate. For a system with an initially high concentration of active sites, the growing fil obtained was quite smooth, whereas when islands of active sites existed a fingerlike unstable, growth mode developed. For the latter system, the memory effects have a high degree of persistence, both in space (because of the formation of islands of active sites) and in time. This long persistence finall causes development of fingerlik structures. However, for the former system, the main cause for the fil smoothness is the high and homogeneous concentration of active sites. It is worth mentioning that in the $\mathrm{SiH}_{4} / \mathrm{O}_{2}$ CVD system, memory effects are consistent with the chemical nature of the deposition process. In such a process, hydrogenated $\mathrm{SiO}_{m} \mathrm{H}_{n}$ species, which are formed in the gas phase, are expected to be the predominant precursors. Once deposited, these species would lead to renewed active sites, such as $\equiv \mathrm{SiH}$, and would be eventually converted into passive sites through different dehydrogenation/passivation mechanisms. ${ }^{28}$

Summarizing our experimental data, it seems that our system could be described as a heterogeneous surface, being composed by two types of sites, active and passive, with 
different sticking probabilities. At $611 \mathrm{~K}$ and $723 \mathrm{~K}$, the concentrations of these species are quite different, leading to different values of the average sticking probability $s$ in agreement with previous reports. ${ }^{12}$ Thus, a complete theoretical model should incorporate these facts, as well as the memory effects mentioned above, in order to account not only for the different surface scaling behaviors obtained at the two temperature values considered, but also for the different fil roughnesses obtained.

\section{DISCRETE MODEL}

In this section, we take the firs step in the formulation of a generalized model which reproduces the experimental observations. We, thus, now consider the simplest discrete growth model which incorporates surface heterogeneity and memory effects. At this point, it is necessary to stress that our aim is not to develop a fully atomistic model accounting for the various growth processes pertinent to the above effects; rather, we wish to describe our system statistically, in terms of distributions of active and passive sites, which may actually change with growth time.

As seen above, our silica film feature both active and passive surface sites. Thus, it is reasonable to assume that memory effects analogous to those in Refs. 18 and 19 may also play an important role in CVD silica growth from silane precursors. However, memory effects of long persistence combined with the formation of permanent islands of active sites should be discarded since, we do not observe fingerlik voided structures. Hence, and although, we have found for our experimental system that there are active sites with varying reactivities, we can assume, within the simplest approximation, that there are only two types of sites, namely, active sites, with local sticking probability $s_{a}$, and passive sites with sticking probability $s_{p} \leqslant s_{a}$. Sites are distributed across the surface with surface coverages $X_{a}$ and $X_{p}=1-X_{a}$, respectively. Both types of sites have relatively short average lifetimes for exchange of site state (active to passive, or vice versa), $\tau_{a}$ and $\tau_{p}$, respectively. On average, for $t>\tau_{j}$ a surface site of type $j=a$ or $p$ has lost the memory of its previous state. Moreover, in principle, the values of $X_{a}$ and $X_{p}$ change with deposition temperature, as observed by IR and Raman spectroscopies. The average sticking probability $s$ is given in this model by $s=s_{a} X_{a}+s_{p} X_{p}$. For such a system, the time evolution of the surface coverages is provided, e.g., by

$$
\frac{d X_{a}}{d t}=-\frac{X_{a}}{\tau_{a}}+\frac{X_{p}}{\tau_{p}},
$$

whose solution is of the form

$$
X_{a}(t)=\tau_{\|} / \tau_{p}+\text { const } \exp \left(-t / \tau_{\|}\right),
$$

where $\tau_{\|}$define as

$$
\tau_{\|}=\left[\frac{1}{\tau_{a}}+\frac{1}{\tau_{p}}\right]^{-1}
$$

is, thus, the natural time scale of the system. ${ }^{29}$ As a consequence, a stationary state is eventually reached for $t \gtrsim 5 \tau_{\|}$, in which $X_{a} \tau_{p}=X_{p} \tau_{a}$.

\section{A. Monte Carlo simulations of random deposition with heterogeneity}

We will next incorporate the above approximate description of memory effects and surface heterogeneity into a simple discrete dynamical model amenable to Monte Carlo type numerical simulations. Specificall, we simulate a random deposition ${ }^{7}$ process with average deposition flu $F$, and populations of active and passive sites as in the previous paragraph, with sticking probabilities $s_{a}$ and $s_{p}$, and surface coverages $X_{a}$ and $X_{p}=1-X_{a}$. Thus, the average growth rate is $v=\Omega F s=\Omega F\left(s_{a} X_{a}+s_{p} X_{p}\right)$, where $\Omega$ is the volume of the aggregating units, which will be set to unity in what follows. As described above, the memory effect is accounted for by including lifetimes $\tau_{a}$ and $\tau_{p}$ for the $a \rightarrow p$ and $p$ $\rightarrow a$ state changes of surface sites, respectively. The specifi implementation of these model features is provided in the Appendix. It is important to recall that for standard homogeneous RD (Ref. 7), the scaling behavior of the surface roughness is as $\sigma \sim t^{1 / 2}$. Figure 5(a) shows the time evolution of the roughness for a system with $\tau_{\|}=0.05\left(\tau_{a}=\tau_{p}=0.1\right)$ and $s=0.5$ for two configurations namely, homogeneous $\left(s_{a}\right.$ $=s_{p}=0.5$ and $\left.X_{a}=X_{p}=0.5\right)$ and heterogeneous $\left(s_{a}=0.8\right.$; $s_{p}=0.2$, and $X_{a}=X_{p}=0.5$ ), and for different values of the flu $F$ (i.e., different growth rates $v$ ). As it is clear from the figure the numerical value of $\sigma$ increases for larger $F$ values, but the temporal scaling behavior (i.e., the slope of the curve) is independent of the flux Moreover, the difference in roughness between the homogeneous and the heterogeneous configuration increases for increasing $F$. That is, the heterogeneous configuratio presents the same scaling behavior as the homogeneous one but with an increased roughness at all times, this effect being more pronounced the larger the product $F \tau_{\|}$is. The physical interpretation is clear. When the effective lifetime $\tau_{\|}$of an arbitrary surface site is shorter than the average time between two successive arrivals of incoming particles at this site, which is proportional to $1 / F$, the site has effectively lost memory, and deposition takes place on a temporally uncorrelated surface. As a consequence, under these conditions there is practically no difference between deposition on a homogeneous and a heterogeneous surface. However, when $\tau_{\|}>1 / F$ the surface site does keep memory of its active or passive state during two consecutive arrivals of particles. Figure 5(b) shows the change of the roughness with deposition time for fixe flu and average sticking coefficien for the homogeneous and heterogeneous configura tions. For the latter, three different cases are shown, corresponding to three different values $\tau_{\|}=0.005,0.05$, and 0.5 . It is clear that for short lifetimes the system behavior (i.e., scaling and numerical roughness) is the same for both configu rations. However, for long enough lifetimes the roughness value of the heterogeneous configuratio is clearly larger than that of the homogeneous configuratio without an appreciable change in the scaling behavior. From these simulations, we can derive important preliminary results. First, it is necessary to have different active species (with different local sticking probabilities) in order to obtain an increase in the roughness. The memory effect by itself is not enough to cause an enhanced roughness. This is clear from Fig. 5, as 



FIG. 5. Monte Carlo simulations of the time evolution of the roughness for a RD system with $\bar{s}=0.5$ for two interface configu rations, namely, homogeneous $\left(s_{a}=s_{p}=0.5\right.$ and $\left.X_{a}=X_{p}=0.5\right)$ and heterogeneous $\left(s_{a}=0.8 ; s_{p}=0.2\right.$ and $\left.X_{a}=X_{p}=0.5\right)$. In panel (a) we fi $\tau_{\|}=0.05\left(\tau_{a}=\tau_{p}=0.1\right)$, while different values of deposition flu $F$ are considered for the homogeneous (solid lines) and hetero geneous (dashed lines) configurations as indicated. Note that the scaling behavior is similar for both configurations but the hetero geneous one shows larger roughness as $F$ increases. In panel (b), we fi $F=10$. The homogeneous configuratio (solid line) is for $\tau_{\|}$ $=0.05$ and for the heterogeneous configuratio three different cases are shown, namely, $\tau_{\|}=0.005(\square), \tau_{\|}=0.05(\bullet)$, and $\tau_{\|}$ $=0.5(\bigcirc)$. All units employed are arbitrary.

the lowest roughness values are always obtained for the homogeneous configuration Thus, two effects, namely, the short-range memory effect and the existence of different species with different sticking coefficients are required to develop an increased roughness without changing the scaling behavior.

\section{CONTINUUM MODELS}

The results obtained in the preceding section, even though corresponding to a very simple model, already provide us with a valuable insight into the mechanisms that underlie the behavior of the roughness in our experimental system. In this section, we exploit this insight in order to formulate a continuum model that will ultimately incorporate relaxation mechanisms on the surface (and thus, as a difference to RD, correlations among different surface sites). This will allow us to account not only for the difference between the numerical values of the roughness for different temperature conditions, but also for the complex scaling behavior observed in Fig. 2, clearly well beyond description by the simple RD model.

\section{A. Continuous time without surface relaxation}

In order to formulate a continuum analog of model (1) that takes into account heterogeneity and memory effects, we start by putting forward a continuum version of the discrete RD model simulated in the preceding section. To this end, we consider the following stochastic continuum model:

$$
\begin{gathered}
\frac{\partial h}{\partial t}=F s(x)+n(x, t), \\
\left\langle n(x, t) n\left(x^{\prime}, t^{\prime}\right)\right\rangle=F s(x) \delta\left(x-x^{\prime}\right) \delta\left(t-t^{\prime}\right) .
\end{gathered}
$$

In the homogeneous case $[s(x)=$ const $\forall x]$, this equation is well known ${ }^{7}$ to provide a correct description of the standard RD model. Note that in our generalized heterogeneous case, the sticking probability $s(x)$ is a site-dependent quantity. Moreover, as in the standard RD case for which the noise strictly follows a Poisson distribution, we have taken the variance of the Gaussian noise $n(x, t)$ in Eqs. (6) equal to the local growth rate $F s(x)$.

Similar to Ref. 30, we now perform a numerical integration of model (6) by a standard Euler scheme ${ }^{17}$ with the additional mutation loop as in the Appendix at each time step (length $\delta t$ ), in which, we reset the state (active/passive) of each surface site according to the corresponding transition probabilities. Results of these simulations are shown in Fig. 6. Active sites have been taken with local sticking probability $s_{a}=1$ and passive sites with sticking probability $s_{p}$ $=0.01$. The lifetime of active sites $\tau_{a}$ has been fixe to 0.1 . We have simulated two system configuration resembling those of our film of $611 \mathrm{~K}(s=0.4)$ and $723 \mathrm{~K}(s=0.08)$. Here, the value of the average sticking probability employed for the low-temperature set has been taken from a linear interpolation from experimental data avaliable for our system. ${ }^{12}$ In order to reproduce the experimental constraint that the growth rate be equal for the two temperature conditions, different values of $F$ have been chosen so that $v$ $=\Omega F s$ is the same. It should be noted that $F$ is not directly accessible in experimental CVD systems, being determined by many other complex factors, such as reactivity of the gas mixture, precursor diffusivity through the stagnant layer, or residence time of the precursor species in the reactor. ${ }^{25}$ However, the total gas flo rate $R_{g}$ allows for an indirect control over these, so that the actual growth rate (i.e., $v=\Omega F s$ ) has the same value for both temperatures, as stated in Sec. II. Thus, in particular, $F$ and $R_{g}$ do not have the same physical meaning. On the other hand, the values of $X_{a}$ for both temperature conditions have been chosen according to the lower concentration of active sites found in the high-temperature set. This choice fixe the ratio $X_{a}(611 \mathrm{~K}) / X_{a}(723 \mathrm{~K})=5.7$ and, accordingly, $X_{p}(723 \mathrm{~K}) / X_{p}(611 \mathrm{~K})=1.56$. These pa- 




FIG. 6. Time evolution of the roughness for model (6) with $\tau_{a}$ $=0.1, s_{a}=1.0$, and $s_{p}=0.01$. Two configuration are considered, resembling those corresponding to the low temperature condition $\left(\bigcirc: F=248.1 ; \bar{s}=0.4, X_{a}=0.403\right.$, and $\left.\tau_{\|}=0.0597\right)$ and high temperature condition $\left(\bullet: F=1250 ; \bar{s}=0.08, X_{a}=0.0707\right.$, and $\tau_{\|}$ $=0.093$ ). The exponent value (slope) $\beta=0.5$ is indicated to show that both systems feature the same scaling behavior. Note the in creased roughness (three times larger) of the high $T$ configuration Units employed for all axes are arbitrary.

rameters imply that the corresponding memory effects last for $\simeq 9 \mathrm{ML}$ and $\simeq 6 \mathrm{ML}$, where $\mathrm{ML}$ stands for monolayer, for the $733-\mathrm{K}$ and $611-\mathrm{K}$ films respectively. Figure 6 shows that both systems present the same scaling behavior (namely, that of standard RD) but the roughness increases for the high-temperature system. Moreover, the ratio $\sigma(723 \mathrm{~K}) / \sigma(611 \mathrm{~K})=3.3$ is of the same order of magnitude as that observed experimentally (see Fig. 2). Thus, in principle, the above choice of the parameters seems consistent with our experimental observations.

\section{B. Continuous time with surface relaxation}

The simulations performed for the continuum model (6) in the preceding section are important because they allow us to generate independent realizations of a stochastic perturbation $\tilde{n}(x, t)$ [define as the whole rhs of Eq. (6a) for each value of $x$ and $t$ ] that features correlations induced by the heterogeneity and memory effects. The next step we will take is to finall add correlations among different surface sites thus far taken as independent by means of appropriate deterministic surface relaxation mechanisms. For this, we can rely on our previous work, ${ }^{10}$ as expressed in the deterministic terms already present in Eq. (1a), whose physical interpretation has been already discussed. Thus, we propose the following stochastic equation for our CVD silica films

$$
\frac{\partial h}{\partial t}=-K \nabla^{4} h+\epsilon \frac{\theta}{\theta}+\frac{\lambda}{2}(\boldsymbol{\nabla} h)^{2}+\tilde{n}(x, t) .
$$

We stress that the noise term $\tilde{n}(x, t)$ is define as the rhs of Eq. (6a) as generated in those simulations, so that, we do not have an analytic expression for its correlations. In particular, $\tilde{n}(x, t)$ does not have, in principle, zero average. A more complete statistical characterization of $\tilde{n}(x, t)$ is presented below in Sec. V C.

Results of our simulations of Eq. (7) are shown in Figs. 7(a) 7(c) for parameter values $L=512, K=1, \epsilon=1$, and $\delta t$ $=0.01$. Three simulations are shown, one including the lowtemperature noise and $\lambda=0.01$ (dashed line), and two other for the high-temperature noise for $\lambda=0.01$ (solid line) and $\lambda=0.04$ (dotted line). Note that the numerical values of the exponents $\alpha$ and $\beta$ depend on dimensionality, and thus differ in the experiments and in our numerical simulations. However, the qualitative type of scaling is the same. ${ }^{11}$ Namely, for the three parameter choices in Fig. 7(a) an unstable region with $\beta \geq 0.5$ is observed for very short times. This zone corresponds to the temporal range characteristic of the memory effects (i.e., $t \leqslant \tau_{\|}$). For the low-temperature system, we fin after this transient a time regime within which $\beta \simeq 0.39$. This value is close to that expected for a growth process determined on a one-dimensional substrate by linear surface diffusion relaxation effects. ${ }^{7,8}$ Finally, for long times the behavior of the roughness for low temperature is described by $\beta \geq 0.5$, indicating unstable growth due to the shadowing term included in Eq. (7). For the hightemperature system, two simulations are presented for two different values of $\lambda$. If $\lambda=0.01$ (solid line), we observe that after the initial transient $t \leqslant \tau_{\|}$a long-time region appears with $\beta \simeq 0.4$. For longer times, $\sigma$ seems to reach the asymptotic KPZ behavior (namely, $\beta=0.33$ ). This effect is more evident in the simulation with $\lambda=0.04$ (dotted line). In this case, initially for $t \gtrsim \tau_{\|}$an unstable region due to the shadowing term dominates growth, but is followed for very long times by KPZ scaling. Two important facts need to be stressed. First, except for $t \leqslant \tau_{\|}$the scaling behavior of Eq. (7) is similar for both temperature conditions to that found experimentally (see Fig. 2) and previously reported. In this respect, Eq. (7) retrieves the experimentally correct scaling behavior already accounted for by model (1). Second, the numerical value of the roughness increases for the hightemperature set (independent of the $\lambda$ value) with respect to the low-temperature set, as is observed experimentally (Fig. 2) but Eq. (1) does not predict. ${ }^{10}$

In order to completely characterize the surface morphologies predicted by Eq. (7), Fig. 7(b) shows the PSD curves obtained from the simulation of Eq. (7) for the same three sets of conditions as in Fig. 7(a). ${ }^{11}$ For the low-temperature set (dashed line), two scaling regions are found. For large $k$ values $\alpha \simeq 1.5$, which again implies that at these length scales surface diffusion relaxation processes are prevalent. ${ }^{7,8}$ For smaller $k$ values $\alpha \simeq 1$, which corresponds to the unstable region observed in Fig. 7(a). For the high-temperature set (both values of $\lambda$ ), the linear surface diffusion region is also observed for larger $k$ values. For smaller $k$ a short intermediate region characterized by $\alpha \simeq 0.8$ is also observed, but for even smaller $k$ values (i.e., larger length scales) the value obtained, $\alpha \simeq 0.5$, is close to the one-dimensional KPZ behavior. Essentially, the same behavior found in Fig. 7(a) is observed, but now the initial surface diffusion region is more clearly observed. Again, larger roughness is obtained for the 

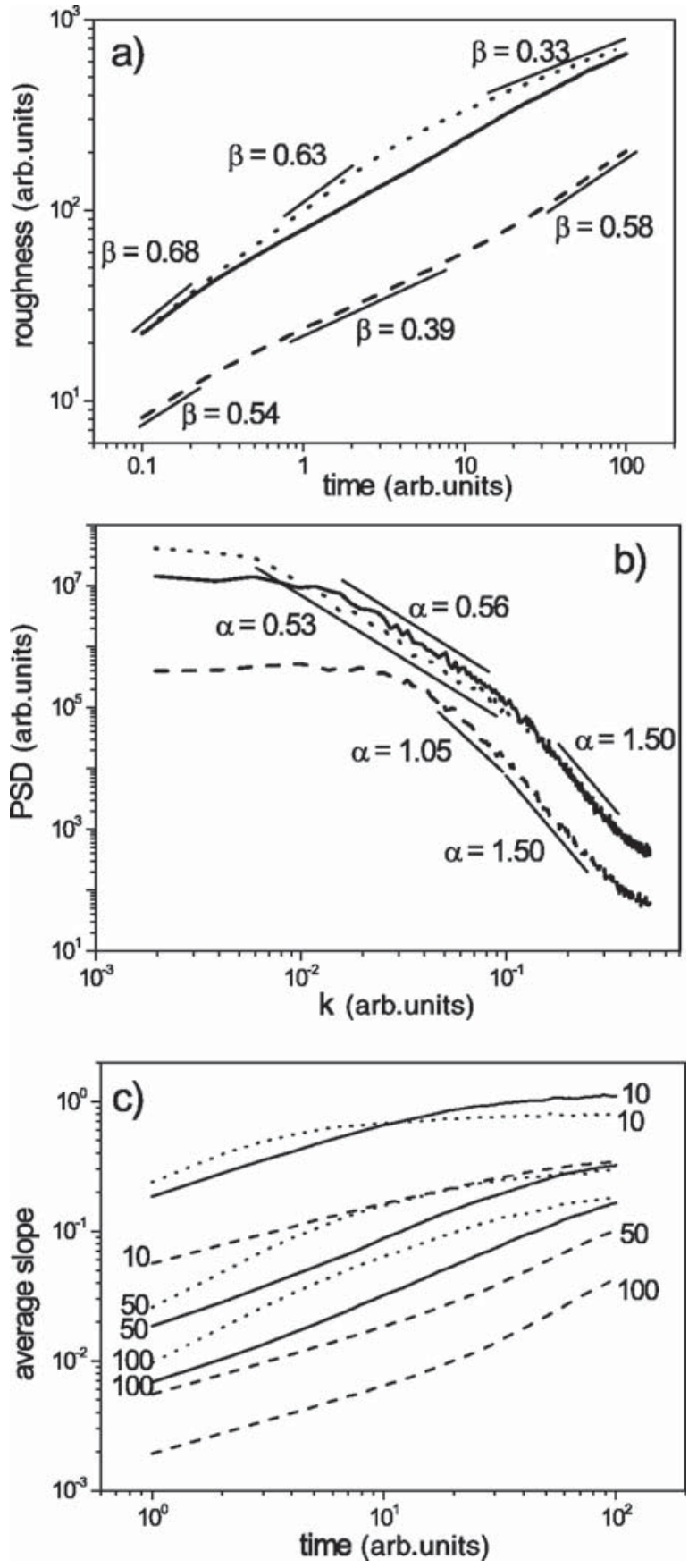

FIG. 7. (a) Time evolution of the interface roughness from a numerical integration of Eq. (7) for $L=512, K=1, \epsilon=1$, and $\delta t$ $=0.01$ for the low $T$ noise configuratio of Fig. 6 with $\lambda=0.01$ (dashed line), and for the high $T$ configuratio of Fig. 6 with $\lambda$ $=0.01$ (solid line) and $\lambda=0.04$ (dotted line). Note the initial un stable regime for both $T$ conditions and the different asymptotic regimes for the low $T$ (unstable) and high $T$ (KPZ) configurations (b) PSD from a numerical integration of Eq. (7) for the same noise conditions and parameter values as in (a). Both in (a) and in (b) relevant exponent values are indicated as the slopes of correspond ing straight lines. (c) Time evolution of the average slope from a numerical integration of Eq. (7) for the same noise conditions and parameter values as in (a), and for different window sizes $\ell$, as indicated. Units employed for all axes are arbitrary.

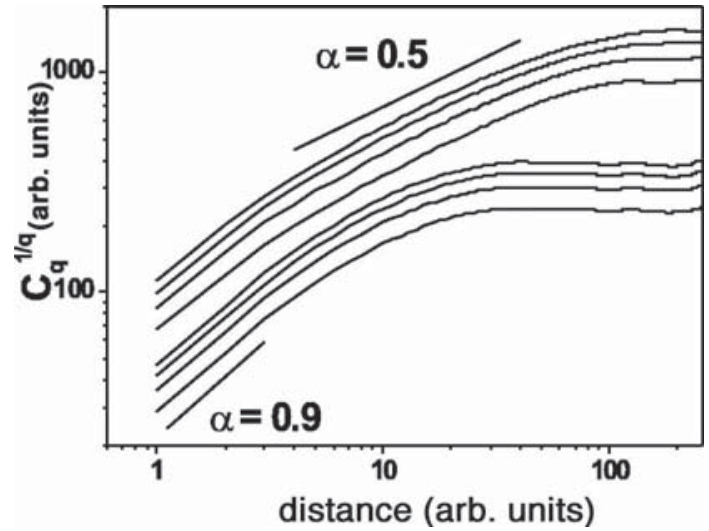

FIG. 8. Logarithmic plots of the $q$ th root of the moments of the height difference distribution $C_{q}(r, t)$ [Eq. (3)] for several values of $q$ from simulations of Eq. (7) for $\lambda=0.04$ (uppermost set of four curves, sequence $q=1,2,3,4$, bottom to top) and $\lambda=0.01$ (lower most set of four curves, sequence $q=1,2,3,4$, bottom to top) at simulation times after saturation. Straight lines are shown for refer ence having slopes, as indicated, that correspond to relevant values of the roughness exponent $\alpha$. All units are arbitrary.

high-temperature set. One detail worth mentioning is that the transient region associated with surface diffusion is smaller for the high-temperature set than for the low-temperature one. This fact was also observed experimentally, but a clear account was not found until now. The surface diffusion transient could not be clearly seen in the experimental plots of $\sigma(t)$ for both temperatures, rather only the unstable and KPZ (for $T=723 \mathrm{~K}$ ) regions could be seen. However, all three regimes were observed in the PSD curves, in agreement with the present simulation results. An additional experimental feature which again is well accounted for by Eq. (7) is the time evolution of the average slope. Results are shown in Fig. 7(c) for $m$ as obtained in simulations of Eq. (7) for the high- $(\lambda=0.04)$ and low- $(\lambda=0.01)$ temperature conditions. This plot compares favorably with the experimental results in Fig. 2. Additionally, and in parallel with our analysis of the experimental data, we have verifie the self-affin character of the simulated surface profiles The results shown in Fig. 8 effectively correspond to a truly self-affin interface in which there is only one independent roughness exponent, again in qualitative agreement with the experimental results.

We can finall compare the results of the simulation of Eq. (7) for the high- and low-temperature conditions with the experimental data in terms of the actual surface morphologies. Figure 9 shows the evolution of the surface profile obtained after the simulations of Eq. (7) employing the high[Fig. 9(a)] and low- [Fig. 9(b)] temperature conditions together with the experimental profil evolution for both temperatures [Figs. 9(c) and 9(d)]. Note that whereas in the simulated profile the time evolution of the position of a given point can be easily followed, this is not the case in the experimental profiles because each AFM snapshot corresponds to a different sample. Note, moreover that the experimental profile correspond to $14-\mu \mathrm{m}$-wide scans of the types of Figs. 1(c) and 1(d), rather than 500-nm-wide scans such as those in Figs. 1(a) and 1(b). For the low-temperature condi- 

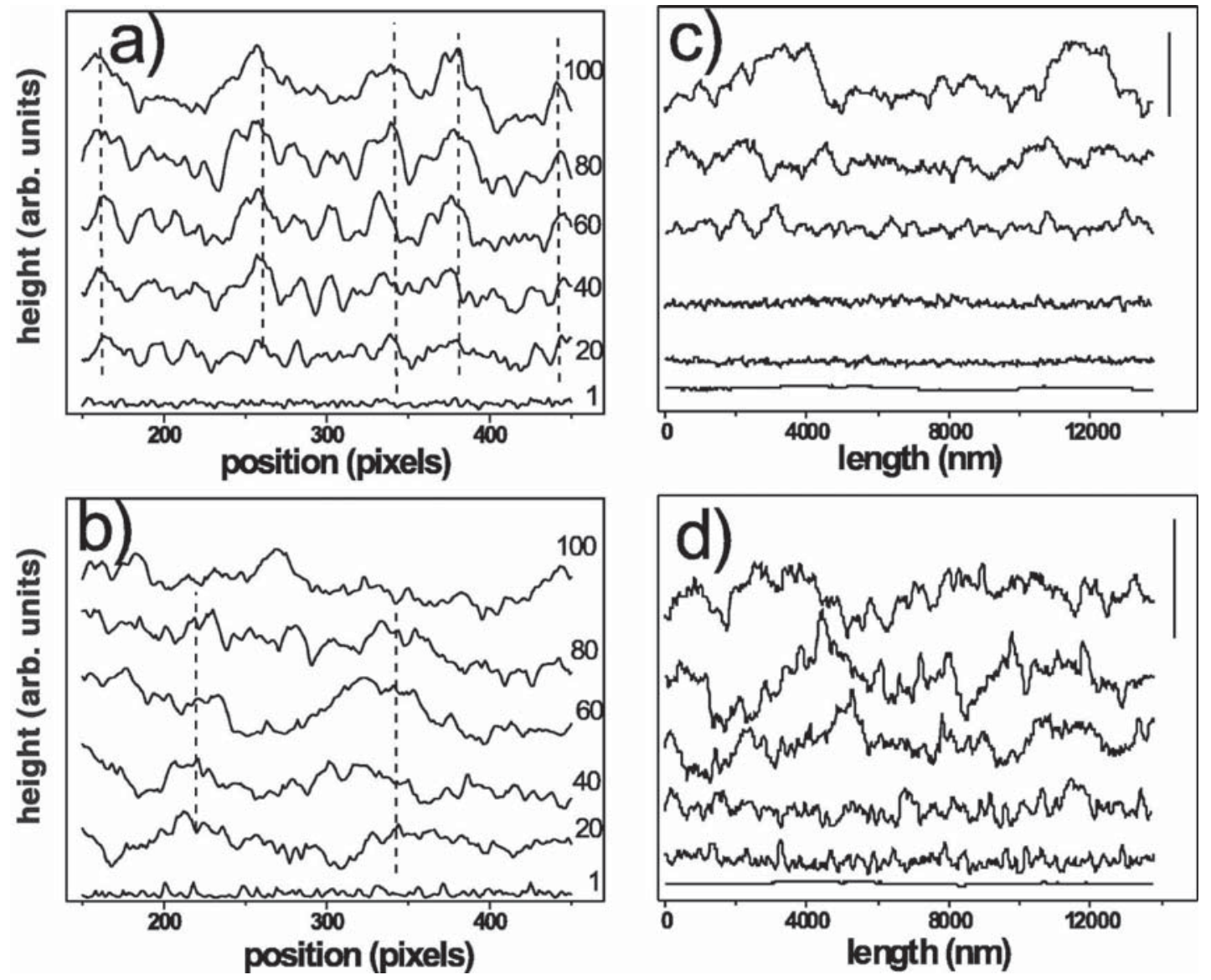

FIG. 9. (a) and (b): Interface profil evolution from numerical integrations of Eq. (7) for $L=512, K=1, \epsilon=1$, and $\delta t=0.01$ for times from $t=1$ up to $t=100$ for the low $T$ configuratio with $\lambda=0.01$ (a) and for the high $T$ configuratio with $\lambda=0.04$ (b). The vertical dashed lines guide the eye along the evolution of various surface protrusions. Units employed are arbitrary. (c) and (d): Experimental profil evolutions for the $611 \mathrm{~K}$ (c) and $723 \mathrm{~K}$ (d) films The solid bar represents $100 \mathrm{~nm}$ (c), and $200 \mathrm{~nm}$ (d), respectively.

tions, the simulated profile are characterized by the development of protrusions [indicated by dashed lines in Fig. 9(a)]. With time, these protrusions persist, growing both vertically and laterally, leading to highly asymmetric profiles The behavior is quite different for the high-temperature conditions [Fig. 9(b)]. Under these conditions, large protrusions can appear (dashed lines) but they grow laterally rather than vertically and eventually disappear, in agreement with the higher relevance of the KPZ term in Eq. (7). Figures 9(c) and (d) show the experimental profil evolution for $611 \mathrm{~K}$ and $723 \mathrm{~K}$ sets. For the low-temperature film large, cauliflowerlik ${ }^{10}$ protrusions develop, which are asymmetric along the growth direction. These features grow both laterally and vertically with deposition time, alike those observed in the simulated profile [see Fig. 9(a)], in particular, for the longest deposition and simulation times. On the contrary, for the high-temperature set the surface profil does not show large protrusions, but rather tends to saturation.

In Fig. 7(a), it was observed that for the initial stages of growth, $t \leqslant \tau_{\|}$, before the surface diffusion mechanism operates, an unstable region develops. This initial region is not shown in the surface profile of Fig. 9(a). Hence, it is interesting to show [Fig. 10(a)] the surface profile for the very early stages of growth when memory effects are predominant. Figure 10(a) shows that for the high-temperature conditions, in which $t \leqslant \tau_{\|}=0.093$, needlelike structures develop. This fast vertical growth is due to the memory effect of the active (i.e., growing) surface sites. However, for $t$ $>\tau_{\|}$when the surface sites have already lost memory, surface features begin to grow also laterally. In Fig. 10(b), we plot the evolution of the surface roughness during the very firs stages of growth for the high-temperature conditions. Clearly, for the time range in which the surface sites have memory of their state $\left(t \leqslant \tau_{\|}\right)$, a large instability $(\beta \simeq 0.9)$ develops, which is reduced for $t>\tau_{\|}$. The shortest deposition time in our experimental system was $5 \mathrm{~min}$. Since, we have not detected this initial unstable regime, it is reasonable to conclude that the memory effect for our system lasts less than $5 \mathrm{~min}$. It is worth mentioning that a memory of 9 and 6 ML was set for the high- and low-temperature conditions in our simulations. These magnitudes led to $\sigma(723 \mathrm{~K})$ $\simeq 3 \sigma(611 \mathrm{~K})$, which is in the same range as the ratio found experimentally. These magnitudes of the memory effect would lead, for the actual $v=20 \mathrm{~nm} / \mathrm{min}$, to average lifetimes clearly shorter than $1 \mathrm{~min}$, in qualitative agreement with the experimental results. 

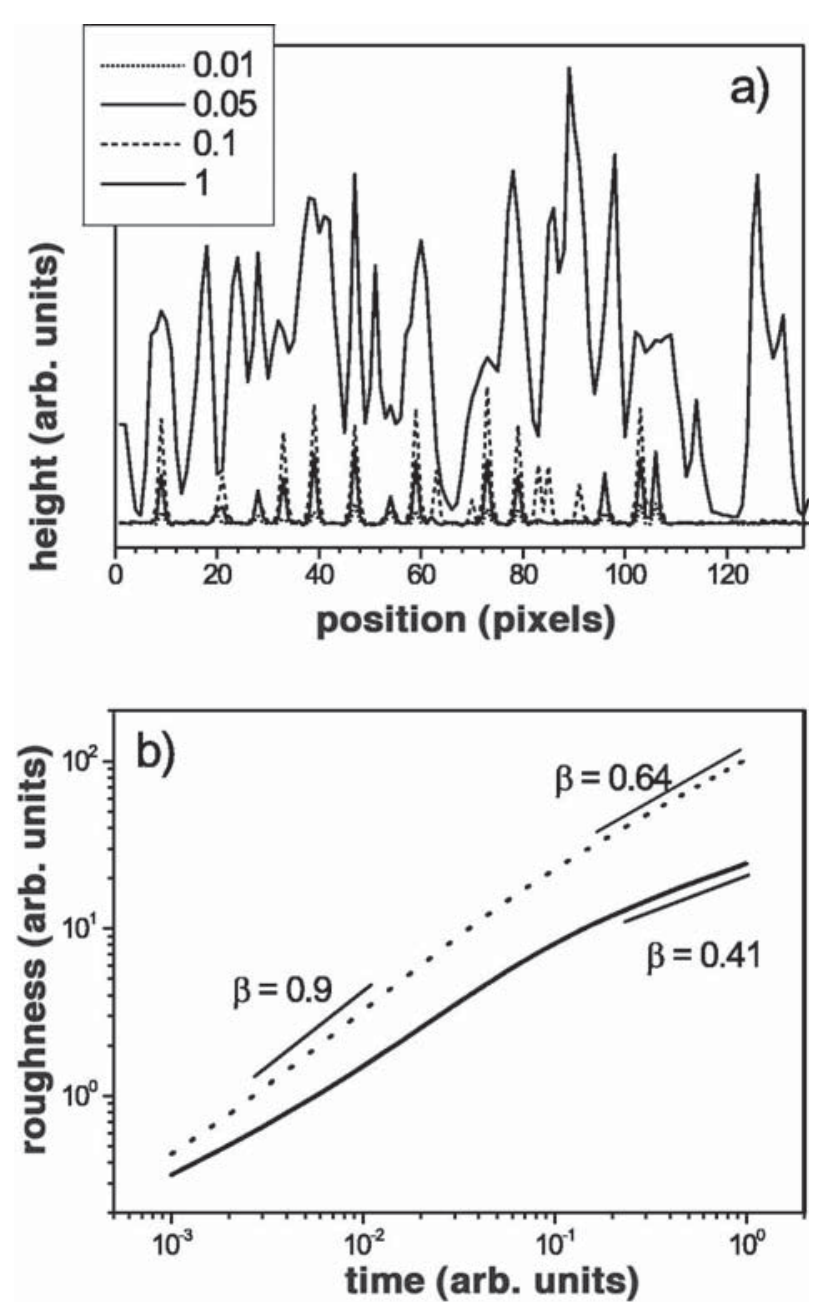

FIG. 10. (a) Initial stages of the interface profil evolution from a numerical integration of Eq. (7) for the high $T$ configuratio with $\delta t=0.001$ and $\lambda=0.04$. Simulation time for each profil is indi cated in the legend. (b) Initial stages of the time evolution of the interface roughness from a numerical integration of Eq. (7) for $\delta t$ $=0.001$ for the low $T$ configuratio with $\lambda=0.01$ (solid line) and for the high $T$ configuratio with $\lambda=0.04$ (dotted line). The values of $\tau_{\|}$for each $T$ configuratio are those of Fig. 6. Units employed for all axes are arbitrary.

\section{Exponentially time correlated noise with surface relaxation}

The analysis in the preceding section consisted in the numerical simulation of our former Eq. (1a), but including the noise obtained from the simulation of Eq. (6), rather than the simple noise, Eq. (1b), previously considered. ${ }^{10}$ This procedure has enabled us to account for the whole set of morphological observations both at high and low temperatures. A natural step now is trying to characterize the noise correlations existing in model (6), and, if possible, to account for them through an appropriate generalization of the continuum model, Eq. (1a). Given that our model of heterogeneity involves populations of active and passive sites that decay according to exponential laws characterized by average lifetimes, a natural guess is ${ }^{17}$ that the correlations of the noise generated in the simulations of model (6) decay exponen-



FIG. 11. Semilogarithmic plots of the autocorrelation function of the high $T(\bigcirc)$ and low $T(\bigcirc)$ noise configuration $\tilde{n}(x, t)$ gen erated by model (6), which have been considered in Figs. 6 and 7. The straight lines show the exponential decay in Eq. (9) with $\rho_{\text {eff }}$ $=0.8788, \quad \tau_{\text {eff }}=0.0871$ (upper), and $\rho_{\text {eff }}=0.079, \quad \tau_{\text {eff }}=0.0544$ (lower). Units employed for all axes are arbitrary.

tially in time, and are otherwise uncorrelated in space. By definin $\tilde{N}(x, t)$ as the fluctuatio of $\tilde{n}(x, t)$ around its spatial average, we thus expect

$$
\left\langle\widetilde{N}(x, t) \tilde{N}\left(x^{\prime}, t^{\prime}\right)\right\rangle=\gamma\left(t-t^{\prime}\right) \delta\left(x-x^{\prime}\right),
$$

where

$$
\gamma(t)=\left(\rho_{\text {eff }} / \tau_{\text {eff }}\right) \exp \left(-|t| / \tau_{\text {eff }}\right),
$$

with constant values of $\rho_{\text {eff }}$ and $\tau_{\text {eff }}$ which are to be determined; $\rho_{\text {eff }}$ determines the roughness value, whereas $\tau_{\text {eff }}$ quantifie the duration of the temporal correlation. In order to assess whether this is an accurate assumption, we have computed the correlations of the noise generated by Eq. (6) for the high- and low-temperature conditions. The results are displayed in Fig. 11. It is clear that the low-temperature noise follows quite closely the anticipated exponential decay (8) and (9), with parameter values $\rho_{\text {eff }}=0.079$ and $\tau_{\text {eff }}$ $=0.0544$. On the contrary, it appears that the hightemperature noise displays two different behaviors, one for short times $(t<0.5)$, where there is exponential decay with $\rho_{\text {eff }}=0.8788$ and $\tau_{\text {eff }}=0.0871$, and another one, for $t>0.5$, where a power-law decay seems to hold. Note that $\rho_{\text {eff }}$ is roughly one order of magnitude larger for the hightemperature condition, in accordance with the larger surface roughness.

In order to probe in more detail the implications of assuming exponentially correlated noise, we simulate model (1), but this time considering a noise $\zeta(x, t)$ with exact time correlations of form (8), namely, we simulate the following continuum model:

$$
\begin{gathered}
\frac{\partial h}{\partial t}=v-K \nabla^{4} h+\epsilon \frac{\theta}{\theta}+\frac{\lambda}{2}(\nabla h)^{2}+\zeta(x, t), \\
\left\langle\zeta(x, t) \zeta\left(x^{\prime}, t^{\prime}\right)\right\rangle=(\rho / \tau) e^{-\left|t-t^{\prime}\right| / \tau} \delta\left(x-x^{\prime}\right),
\end{gathered}
$$



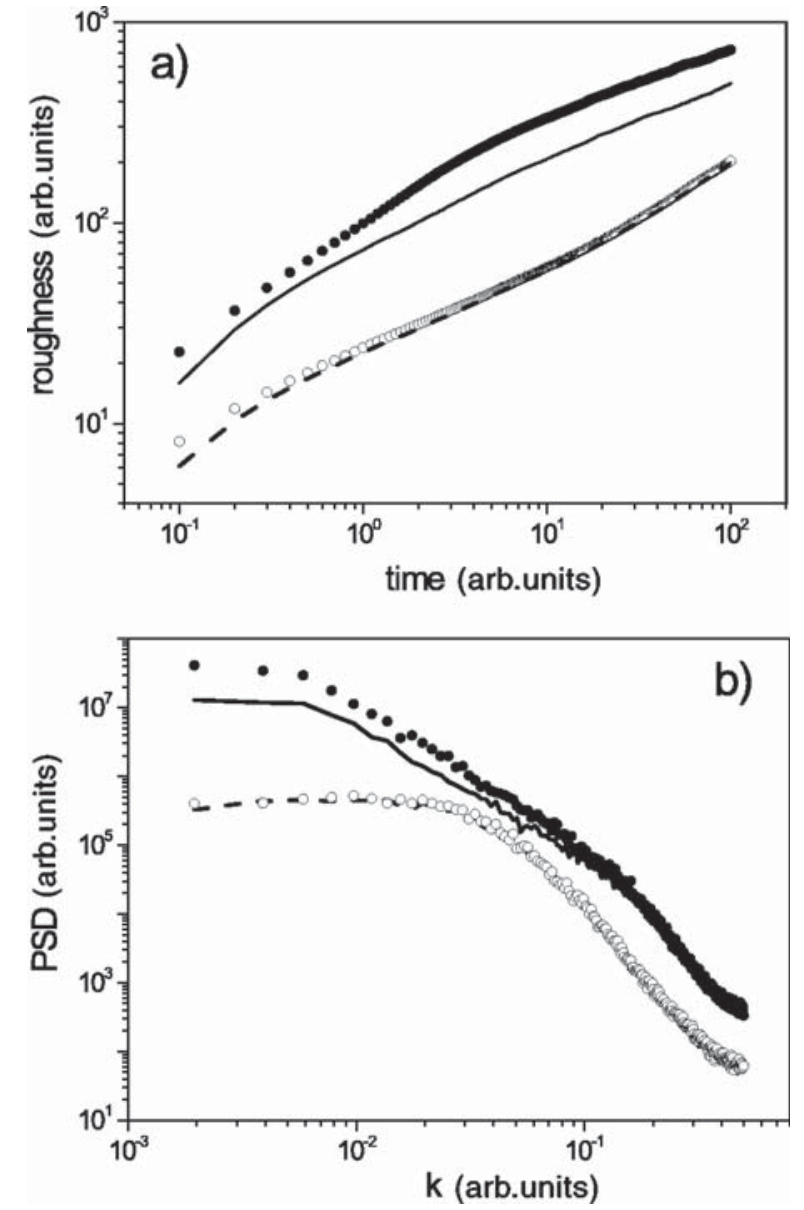

FIG. 12. (a) Time evolution of the interface roughness from a numerical integration of Eqs. (7) and (10) for $L=512, K=1, \epsilon$ $=1$, and $\delta t=0.01$, for the low $T$ configuratio of Fig. 6 with $\lambda$ $=0.01$ (open circles and dashed line, respectively), and for the high $T$ configuratio of Fig. 6 with $\lambda=0.04$ (solid circles and solid line, respectively). (b) PSD from a numerical integration of Eqs. (7) and (10) for $L=512, K=1, \epsilon=1$, and $\delta t=0.01$ for the low $T$ con figuratio with $\lambda=0.01$ (open circles and dashed line, respectively), and for the high $T$ configuratio with $\lambda=0.04$ (solid circles and solid line, respectively). In both panels, there is quantitative agree ment between Eqs. (7) and (10) for the low $T$ systems, whereas for the high $T$ conditions the agreement is restricted to the scaling be havior. Units employed for all axes are arbitrary.

where $\rho$ and $\tau$ are constants. In Figs. 12(a) and 12(b) we show both the roughness and the PSD thus obtained for the high- and low-temperature cases, and compare them with the results previously obtained in Fig. 7 for the simulation of model (7). Not surprisingly after the results in Fig. 11, the results for models (7) and (10) almost overlap for the lowtemperature condition. However, for the high-temperature set the results for these two continuum models do not match, although the scaling behavior is very similar (with respect to the sequence: noise instability surface diffusion scaling shadowing instability KPZ scaling). We attribute the origin of such discrepancy to the different correlation functions of the noise distributions $\widetilde{N}(x, t)$ and $\zeta(x, t)$ in the hightemperature conditions, in particular, due to the power-law tail for the time correlations of $\tilde{N}(x, t)$. Note that the hightemperature condition in Sec. V B features a memory parameter $\tau_{\|}$that is roughly $50 \%$ larger than that of the lowtemperature condition, and hence the characterization of the noise correlations by an exponential decay law may be inappropriate.

\section{SUMMARY AND CONCLUSIONS}

We have shown that the mere dependence of the sticking probability with temperature does not suffic to fully describe the growth of silica film by CVD at $611 \mathrm{~K}$ and $723 \mathrm{~K}$. Rather, it is necessary to consider the fact that this system presents surface sites with both high (active sites) and low (passive sites) reactivity for growth, the concentration of active (passive) sites being relatively low (high) for the hightemperature film in comparison with that of the lowtemperature set. Once this is taken into account, together with the assumption of a short-range memory effect (i.e., an average lifetime), for each type of surface site, a complete theoretical description can be provided that accounts not only for the experimentally observed scaling behavior but also for the observation of a larger roughness for the hightemperature films This improvement in the theoretical modeling over the current description ${ }^{10}$ is achieved by keeping the same deterministic relaxation terms in the phenomenological interface equation proposed in Ref. 10, but including short-range temporally correlated noise, since the memory and heterogeneity effects determine the noise intensity and correlation time $\tau$. From the point of view of modeling surface growth, we have proposed a generalization of the random deposition model that takes into account (i) a finit flu of particles; (ii) two populations of surface sites identifie by two different values of the sticking probabilities, with transitions between the two possible states. We have also proposed a continuum description of this model, which, we have seen numerically to be approximately described by a stochastic equation with a more standard noise that is exponentially correlated in time.

\section{ACKNOWLEDGMENTS}

This work has been performed within the CONICETCSIC research program, and has been partially supported by Programa de Cooperación Científic con Iberoamérica (MEC), CAM Grant No. 7220-ED/082, and DGES Grants Nos. MAT97-0698-C04, PB96-0119, and BFM2000-0006. F.O. acknowledges financia support from CAM.

\section{APPENDIX: RANDOM DEPOSITION WITH HETEROGENEITY}

The simulation steps of the RD model with heterogeneity, which has been considered in Sec. IV A are as follows. Initially, we take a fla interface $h(\mathbf{r}, t=0)=0 \forall \mathbf{r}$, with $\mathbf{r}$ being an integer number (substrate dimensionality is immaterial, all sites being independent of one another). Also at $t=0$, we distribute randomly active and passive sites with coverages 
$X_{a}$ and $X_{p}$, respectively. At any given time, we choose randomly a surface site (topmost occupied $h$ value at a given substrate position $\mathbf{r})$. We grow on that site $[h(\mathbf{r}, t+\Delta t)$ $=h(\mathbf{r}, t)+1]$ with the corresponding sticking probability $s(\mathbf{r}, t)$, and run a mutation loop in which, we change the state of each surface site according to the transition probabilities $\mathcal{P}_{a \rightarrow p}=\Delta t / \tau_{a}$ and $\mathcal{P}_{p \rightarrow a}=\Delta t / \tau_{p}$. Here, $\Delta t=1 /\left(N_{0} F\right)$, with $F$ the flu and $N_{0}$ the total number of surface sites.
*Present address: teCnAtom, SA, Avenida Montes de Oca 1, E 28709 San Sebastián de los Reyes, Spain. Electronic address: fojeda@tecnatom.es

†Electronic address: cuerno@math.uc3m.es

Electronic address: robsalva@inifta.unlp.edu.ar

${ }^{\S}$ Electronic address: far@icmm.csic.es

"Electronic address: 1vb@icmm.csic.es

${ }^{1}$ K. F. Jensen and W. Kern, in Thin Film Processes II, edited by J. L. Vossen and W. Kern (Academic, Boston, 1991).

${ }^{2}$ C.H.J. Van den Brekel and A.K. Jansen, J. Cryst. Growth 43, 364 (1977).

${ }^{3}$ B.J. Palmer and R.G. Gordon, Thin Solid Films 158, 313 (1988)

${ }^{4}$ G.S. Bales, A.C. Redfield and A. Zangwill, Phys. Rev. Lett. 62, 776 (1989).

${ }^{5}$ G.S. Bales and A. Zangwill, Phys. Rev. Lett. 63, 692 (1989); J. Vac. Sci. Technol. A 9, 145 (1991).

${ }^{6}$ M.J. Cooke and G. Harris, J. Vac. Sci. Technol. A 7, 3217 (1989).

${ }^{7}$ A. L. Barabási and H. E. Stanley, Fractal Concepts in Surface Growth (Cambridge University Press, Cambridge, 1995).

${ }^{8}$ J. Krug, Adv. Phys. 46, 139 (1997).

${ }^{9}$ F. Family and T. Vicsek, J. Phys. A 18, L75 (1985).

${ }^{10}$ F. Ojeda, R. Cuerno, R. Salvarezza, and L. Vázquez, Phys. Rev. Lett. 84, 3125 (2000).

${ }^{11}$ Note that both in the experimental and theoretical results, the existence of crossover effects implies ranges for the various scaling behaviors that are shorter than desirable in order to ob tain the critical exponents with high accuracy. It is mostly due to the agreement between experiments and models that, we can attribute the various intermediate scaling regions to specifi physical mechanisms and their representation as different terms in the continuum interface equations.

${ }^{12}$ K. Watanabe and H. Komiyama, J. Electrochem. Soc. 137, 1222 (1990).

${ }^{13}$ M. Kardar, G. Parisi, and Y. C. Zhang, Phys. Rev. Lett. 56, 889
(1986).

${ }^{14}$ W.W. Mullins, J. Appl. Phys. 28, 333 (1957).

${ }^{15}$ R.P.U. Karunasiri, R. Bruinsma, and J. Rudnick, Phys. Rev. Lett. 62, 788 (1989).

${ }^{16}$ J.H. Yao, C. Roland, and H. Guo, Phys. Rev. A 45, 3903 (1992); J.H. Yao and H. Guo, Phys. Rev. E 47, 1007 (1993).

${ }^{17}$ J. García Ojalvo and J. M. Sancho, Noise in Spatially Extended Systems (Springer, New York, 1999).

${ }^{18}$ K. Tsukamoto, D. Cheng, H. Komiyama, Y. Nishimoto, N. Toku masu, and K. Maeda, Jpn. J. Appl. Phys., Part 2 38, L68 (1999).

${ }^{19}$ D. Cheng, K. Tsukamoto, H. Komiyama, Y. Nishimoto, N. Toku masu, and K. Maeda, J. Appl. Phys. 85, 7140 (1999).

${ }^{20}$ K. Sneppen, J. Krug, M.H. Jensen, C. Jayaprakash, and T. Bohr, Phys. Rev. A 46, R7351 (1992).

${ }^{21}$ R. Cuerno and A. L. Barabási, Phys. Rev. Lett. 74, 4746 (1995); S. Park, B. Kahng, H. Jeong, and A. L. Barabási, ibid. 83, 3486 (1999).

${ }^{22}$ R. K. Iler, The Chemistry of Silica (Wiley, New York, 1979).

${ }^{23}$ T.A. Michalske and B.C. Bunker, J. Appl. Phys. 56, 2686 (1984).

${ }^{24}$ Q. Sun, J.Z. Yu, L. Zhou, Z.Q. Li, Z. Tang, K. Ohno, and Y. Kawazoe, Europhys. Lett. 43, 47 (1998).

${ }^{25}$ F. Ojeda, I. Montero, F. Abel, and J.M. Albella, Chem. Mater. 13, 3986 (2001); F. Ojeda, Ph.D. thesis, Universidad Autónoma de Madrid, 1999

${ }^{26}$ C.J. Brinker, D.R. Tallant, E.P. Roth, and C.S. Ashley, J. Non Cryst. Solids 82, 117 (1986).

${ }^{27}$ M.E. Bartram and H.K. Moffat, J. Vac. Sci. Technol. A 14, 872 (1996).

${ }^{28}$ F. Ojeda, F. Abel, and J.M. Albella, J. Phys. Chem. B 106, 6258 (2002).

${ }^{29} \mathrm{~W}$. Horsthemke and R. Lefever, Noise Induced Transitions (Springer, New York, 1984).

${ }^{30}$ C. Lam, L.M. Sander, and D.E. Wolf, Phys. Rev. A 46, R6128 (1992). 